\title{
On a class of Darboux-integrable semidiscrete equations
}

\author{
Kostyantyn Zheltukhin ${ }^{1 *}$, Natalya Zheltukhina ${ }^{2}$ and Ergun Bilen ${ }^{1}$
}

\author{
"Correspondence: \\ zheltukh@metu.edu.tr \\ 'Department of Mathematics, \\ Middle East Technical University, \\ Universiteler Mahallesi, Dumlupinar \\ Bulvari 1, Ankara, 06800, Turkey \\ Full list of author information is \\ available at the end of the article
}

\begin{abstract}
We consider a classification problem for Darboux-integrable hyperbolic semidiscrete equations. In particular, we obtain a complete description for a special class of equations admitting four-dimensional characteristic $x$-rings and two-dimensional characteristic $n$-rings. For all described equations, the corresponding $x$ - and $n$-integrals are constructed.
\end{abstract}

Keywords: semidiscrete equations; Darboux integrability; characteristic rings

\section{Introduction}

Classification problems play an important role in the study of integrable equations. For classification of hyperbolic equations, it is convenient to define integrability in terms of characteristic rings. The notion of a characteristic ring was introduced by Shabat for integrable hyperbolic equations of exponential type (see $[1,2])$ and then used by Zhiber to study general integrable hyperbolic equations (see [3-7]). Later, Habibullin extended this notion to the case of semidiscrete and discrete equations (see [8-16]). For more details on characteristic rings, see survey paper [17].

We consider semidiscrete hyperbolic equations that admit nontrivial $x$ - and $n$-integrals, so-called Darboux-integrable equations [18]. It was proved in [9] that a semidiscrete hyperbolic equation is Darboux integrable if and only if its characteristic $x$ - and $n$-rings are finite-dimensional. Description of all equations with characteristic $x$ - and $n$-rings of finite dimensions is a very difficult classification problem. The majority of known Darbouxintegrable semidiscrete equations possess $x$ - and $n$-rings of dimensions not exceeding five (see $[14,16,19])$. Necessary and sufficient conditions for a characteristic $x$-ring to be fourdimensional were obtained in [20] (also see [21] for a characterization of five-dimensional characteristic $x$-rings). In [12] the conditions for a two-dimensional characteristic $n$-ring were obtained. We use these conditions to explicitly derive integrable equations with fourdimensional characteristic $x$-rings and two-dimensional characteristic $n$-rings.

Consider the equation

$$
t_{1 x}=f\left(x, t, t_{1}, t_{x}\right)
$$

where the function $t(n, x)$ depends on the discrete variable $n$ and continuous variable $x$. We use the notations $t_{x}=\frac{\partial}{\partial x} t$ and $t_{1}=t(n+1, x)$. It is also convenient to denote $t_{[k]}=\frac{\partial^{k}}{\partial x^{k}} t$,

(c) The Author(s) 2017. This article is distributed under the terms of the Creative Commons Attribution 4.0 International License (http://creativecommons.org/licenses/by/4.0/), which permits unrestricted use, distribution, and reproduction in any medium, provided you give appropriate credit to the original author(s) and the source, provide a link to the Creative Commons license, and indicate if changes were made. 
$k \in \mathbb{N}$, and $t_{m}=t(n+m, x), m \in \mathbb{Z}$. It was proved in [20] that if equation (1) has a fourdimensional characteristic $x$-ring, then the function $f$ has the form

$$
f=A\left(x, t, t_{1}\right) M\left(x, t, t_{x}\right)+B\left(x, t, t_{1}\right) t_{x}+C\left(x, t, t_{1}\right) .
$$

In this work, we assume that the function $M$ depends only on $t_{x}$ and $f$ does not depend on $x$, that is, we study equations of the form

$$
t_{1 x}=A\left(t, t_{1}\right) M\left(t_{x}\right)+B\left(t, t_{1}\right) t_{x}+C\left(t, t_{1}\right) .
$$

It turns out that we have to consider two cases of $f$ linear and nonlinear in $t_{x}$. The results of our investigation are given in the following theorems.

Theorem 1 Let $f$ be a linear function of $t_{x}$. Equation (3) has a four-dimensional characteristic $x$-ring and a two-dimensional characteristic n-ring if and only if

$$
f=\frac{\gamma(t)}{\gamma\left(t_{1}\right)} t_{x}-\frac{\gamma(t)}{\gamma\left(t_{1}\right)} \sigma(t)+\sigma\left(t_{1}\right)
$$

where the functions $\gamma$ and $\sigma$ satisfy either of the relations

$$
(\gamma(t) \sigma(t))^{\prime}=\gamma(t) \sqrt{B_{1}+B_{2} \gamma(t) \sigma(t)}
$$

or

$$
(\gamma(t) \sigma(t))^{\prime}=\gamma(t) \sqrt{B_{1}+B_{2}(\gamma(t) \sigma(t))^{2}}
$$

with arbitrary constants $B_{1}$ and $B_{2}$.

Theorem 2 Letf be a nonlinear function of $t_{x}$. Equation (3) has a four-dimensional characteristic $x$-ring and a two-dimensional characteristic n-ring if and only if

$$
f=\frac{c_{1} \eta(t) \eta\left(t_{1}\right)}{t_{x}} \text { or } f=\frac{c_{1} e^{c_{2}\left(t+t_{1}\right)}}{t_{x}+P}-P,
$$

where $c_{1}, c_{2}$, and $P$ are arbitrary constants, and $\eta$ is an arbitrary function of one variable, or

$$
f=\sqrt{B^{2}-1} \sqrt{t_{x}^{2}+P t_{x}+Q}+B t_{x}+\frac{1}{2} P(B-1),
$$

where $B, P$, and $Q$ are arbitrary constants.

The paper is organized as follows. First, we give proofs of Theorems 1 and 2, and in the last section, we provide $x$ - and $n$-integrals for equations found in Theorems 1 and 2 . 


\section{Proofs of Theorem 1 and Theorem 2}

\subsection{Preliminary results}

In what follows, all calculations are done on the set of solutions of equation (1), that is, we consider $\ldots, t_{-1}, t_{0}, t_{1}, \ldots$ and $t_{x}, t_{x x}, t_{x x x}, \ldots$ as independent dynamical variables. The derivatives of $\ldots, t_{-1}, t_{0}, t_{1}, \ldots$ and shifts of $t_{x}, t_{x x}, t_{x x x}, \ldots$ are expressed in terms of the dynamical variables using (1).

Let us formulate necessary and sufficient conditions so that equation (3) has a characteristic $x$-ring of dimension four and a characteristic $n$-ring of dimension two. First, we consider the $n$-ring. The following theorem was proved in [12].

Theorem 3 Equation (1) has a characteristic n-ring of dimension two if and only if

$$
D\left(\frac{f_{t}}{f_{t_{x}}}\right)=-f_{t_{1}}
$$

where $D$ is the shift operator: $D g(n, x)=g(n+1, x)$.

We remark that equality (4) implies that

$$
\frac{\partial}{\partial t_{1}}\left(\frac{f_{t}}{f_{t_{x}}}\right)=0
$$

since $f_{t_{1}}$ does not depend on $t_{2}$. We use this observation later.

For the characteristic $x$-ring, we have to consider two cases: $f_{t_{x} t_{x}}=0$, that is, $f$ is a linear function of $t_{x}$, and $f_{t_{x} t_{x}} \neq 0$, that is, $f$ is a nonlinear function of $t_{x}$.

The following theorems were proved in [20].

Theorem 4 Equation (1) with $f_{t_{x} t_{x}}=0$ has a characteristic ring $L_{x}$ of dimension four if and only if

$$
D\left(\frac{K(m)}{m}-m+\frac{f_{t}}{f_{t_{x}}}\right)=\frac{K(m)}{m}+m-f_{t_{1}}
$$

where $K$ is the vector field

$$
\begin{aligned}
K & =\frac{\partial}{\partial x}+t_{x} \frac{\partial}{\partial t}+f \frac{\partial}{\partial t_{1}}+\cdots, \\
\text { and } m & =\frac{-\left(f_{x t_{x}}+t_{x} f_{x_{x}}+f f t_{t_{x}}\right)+f_{t}+f_{t_{x}} f_{t_{1}}}{f_{t_{x}}} .
\end{aligned}
$$

Theorem 5 Equation (1) with $f_{t_{x} t_{x}} \neq 0$ has a characteristic ring $L_{x}$ of dimension four if and only if

$$
D\left(\frac{f_{t_{x} t_{x} t_{x}}}{f_{t_{x} t_{x}}}\right)=\frac{f_{t_{x} t_{x} t_{x}} f_{t_{x}}-3 f_{t_{x} t_{x}}^{2}}{f_{t_{x} t_{x}} f_{t_{x}}^{2}}
$$

and

$$
D \tilde{m}=\tilde{m} f_{t_{x}}-\left(f_{x}+t_{x} f_{t}+f_{t_{1}} f\right)
$$

where $\tilde{m}=\frac{f_{x t_{x}}+t_{x} f_{t_{x}}+f f_{t_{t} t_{1}}-f_{t}-f_{t_{x}} f_{t_{1}}}{f_{t_{x} t_{x}}}$. 
In the same way as in equation (5), we have

$$
\frac{\partial}{\partial t_{1}} m=0 \quad \text { and } \quad \frac{\partial}{\partial t_{1}} \tilde{m}=0
$$

For convenience of the reader, let us give definitions of $x$ - and $n$-integrals and of Darboux-integrable semidiscrete equations.

Definition 6 A function $F\left(x, t, t_{1}, \ldots, t_{k}\right)$ is called an $x$-integral of equation (1) if

$$
D_{x} F\left(x, t, t_{1}, \ldots, t_{k}\right)=0
$$

for all solutions of (1). Here $D_{x}$ is the operator of total differentiation with respect to $x$ : $D_{x} g(n, x)=(d / d x) g(n, x)$.

A function $G\left(x, t, t_{x}, \ldots, t_{[m]}\right)$ is called an $n$-integral of equation (1) if

$$
D G\left(x, t, t_{x}, \ldots, t_{[m]}\right)=G\left(x, t, t_{x}, \ldots, t_{[m]}\right)
$$

for all solutions of (1).

Equation (1) is called Darboux integrable if it admits a nontrivial $x$-integral and a nontrivial $n$-integral.

\subsection{Proof of Theorem 1}

We assume that $f$ is a linear function of $t_{x}$. Thus

$$
f\left(t, t_{1}, t_{x}\right)=A\left(t, t_{1}\right) t_{x}+B\left(t, t_{1}\right)
$$

and equation (1) becomes

$$
t_{1 x}=A\left(t, t_{1}\right) t_{x}+B\left(t, t_{1}\right) .
$$

The proof of the Theorem 1 is based on the following lemmas.

Lemma 7 Let $f_{t_{x} t_{x}}=0$. Then the characteristic n-ring of equation (11) has dimension two if and only if

$$
f\left(t, t_{1}, t_{x}\right)=\frac{c_{1} \gamma(t)}{\gamma\left(t_{1}\right)} t_{x}+\frac{c_{2}}{\gamma\left(t_{1}\right)}-\frac{c_{1} \gamma(t) \sigma(t)}{\gamma\left(t_{1}\right)}+\sigma\left(t_{1}\right),
$$

where $\gamma$ and $\sigma$ are functions of one variable, and $c_{1}, c_{2}$ are constants.

Proof It follows from condition (4) that

$$
\frac{f_{t}}{f_{t_{x}}}=\frac{A_{t}}{A} t_{x}+\frac{B_{t}}{A}
$$

does not depend on $t_{1}$. Hence $\frac{A_{t}}{A}$ and $\frac{B_{t}}{A}$ do not depend on $t_{1}$. So we can write $A\left(t, t_{1}\right)=$ $\gamma(t) \varphi\left(t_{1}\right)$ and $B\left(t, t_{1}\right)=l(t) \varphi\left(t_{1}\right)+\sigma\left(t_{1}\right)$ for some functions $\gamma, \varphi$, and $\sigma$. The function $f$ takes 
form

$$
f\left(t, t_{1}, t_{x}\right)=\gamma(t) \varphi\left(t_{1}\right) t_{x}+l(t) \varphi\left(t_{1}\right)+\sigma\left(t_{1}\right)
$$

Applying condition (4) to $f$ given by (14), we get

$$
\begin{aligned}
& \frac{\gamma^{\prime}\left(t_{1}\right)}{\gamma\left(t_{1}\right)}\left(\gamma(t) \varphi\left(t_{1}\right) t_{x}+l(t) \varphi\left(t_{1}\right)+\sigma\left(t_{1}\right)\right)+\frac{l^{\prime}\left(t_{1}\right)}{\gamma\left(t_{1}\right)} \\
& =-\gamma(t) \varphi^{\prime}\left(t_{1}\right) t_{x}-l(t) \varphi^{\prime}\left(t_{1}\right)-\sigma^{\prime}\left(t_{1}\right) .
\end{aligned}
$$

By comparing the coefficients of $t_{x}$ in (15) we get

$$
\frac{\gamma^{\prime}\left(t_{1}\right)}{\gamma\left(t_{1}\right)}+\frac{\varphi^{\prime}\left(t_{1}\right)}{\varphi\left(t_{1}\right)}=0
$$

so that $\varphi\left(t_{1}\right)=\frac{c_{1}}{\gamma\left(t_{1}\right)}$, where $c_{1}$ is some constant. Substituting this $\varphi$ into equation (15) and collecting the terms independent of $t_{x}$, we get

$$
\gamma^{\prime}\left(t_{1}\right) \sigma\left(t_{1}\right)+\gamma\left(t_{1}\right) \sigma^{\prime}\left(t_{1}\right)+l^{\prime}\left(t_{1}\right)=0
$$

Solving (16), we find

$$
l(t)=-\gamma(t) \sigma(t)+\tilde{c}_{2},
$$

where $c_{2}$ is some constant. Substituting $\varphi$ and $l$ found into equation (14), we get equation (12). We can check that condition (4) is satisfied for function (12).

Now we can rewrite equation (11) as

$$
t_{1 x}=\frac{c_{1} \gamma(t)}{\gamma\left(t_{1}\right)} t_{x}+\frac{c_{2}}{\gamma\left(t_{1}\right)}-\frac{c_{1} \gamma(t) \sigma(t)}{\gamma\left(t_{1}\right)}+\sigma\left(t_{1}\right)
$$

where $\gamma$ and $\sigma$ are functions of one variable, and $c_{1}, c_{2}$ are constants. The equation can be simplified by introducing the new variable

$$
\tau=L(t)
$$

where $L$ satisfies $L^{\prime}(t)=\gamma(t)$. Equation (18) becomes

$$
\tau_{1_{x}}=c_{1} \tau_{x}+c_{2}-c_{1} Q(\tau)+Q\left(\tau_{1}\right)
$$

for some function $Q$ of one variable. We can check that condition (4) is satisfied for the new equation. Hence our change of variable does not affect the dimension of the characteristic $n$-ring.

In the next lemma, we give conditions for equation (20) to have a four-dimensional characteristic $x$-ring. 
Lemma 8 Equation (20) has a four-dimensional characteristic $x$-ring if and only if

$$
Q(\tau)=A_{1} \tau^{2}+A_{2} \tau \quad \text { or } \quad Q(\tau)=A_{1} e^{\alpha \tau}+A_{2} e^{-\alpha \tau}
$$

for some constants $A_{1}, A_{2}$, and $\alpha$.

Proof Applying condition (6) to function $f=c_{1} \tau_{x}+c_{2}-c_{1} Q(\tau)+Q\left(\tau_{1}\right)$, we get

$$
\begin{aligned}
& \frac{c_{1} \tau_{x}+c_{2}-c_{1} Q(\tau)+Q\left(\tau_{1}\right)}{Q^{\prime}\left(\tau_{2}\right)-Q^{\prime}\left(\tau_{1}\right)}\left(c_{1} Q^{\prime \prime}\left(\tau_{2}\right)-Q^{\prime \prime}\left(\tau_{1}\right)\right) \\
& \quad+\frac{Q^{\prime \prime}\left(\tau_{2}\right)\left(c_{2}-c_{1} Q\left(\tau_{1}\right)+Q\left(\tau_{2}\right)\right)}{Q^{\prime}\left(\tau_{2}\right)-Q^{\prime}\left(\tau_{1}\right)}-Q^{\prime}\left(\tau_{2}\right)+Q^{\prime}\left(\tau_{1}\right) \\
& =\frac{c_{1} Q^{\prime \prime}\left(\tau_{1}\right)-Q^{\prime \prime}(\tau)}{Q^{\prime}\left(\tau_{1}\right)-Q^{\prime}(\tau)} \tau_{x}+\frac{Q^{\prime \prime}\left(\tau_{1}\right)\left(c_{2}-c_{1} Q(\tau)+Q\left(\tau_{1}\right)\right)}{Q^{\prime}\left(\tau_{1}\right)-Q^{\prime}(\tau)}+Q^{\prime}\left(\tau_{1}\right)-Q^{\prime}(\tau) .
\end{aligned}
$$

By comparing the coefficients of $\tau_{x}$ in this equality, we get

$$
c_{1} D\left(\frac{c_{1} Q^{\prime \prime}\left(\tau_{1}\right)-Q^{\prime \prime}(\tau)}{Q^{\prime}\left(\tau_{1}\right)-Q^{\prime}(\tau)}\right)=\frac{c_{1} Q^{\prime \prime}\left(\tau_{1}\right)-Q^{\prime \prime}(\tau)}{Q^{\prime}\left(\tau_{1}\right)-Q^{\prime}(\tau)}
$$

which implies that either $c_{1}=1$ and $\frac{c_{1} Q^{\prime \prime}\left(\tau_{1}\right)-Q^{\prime \prime}(\tau)}{Q^{\prime}\left(\tau_{1}\right)-Q^{\prime}(\tau)}$ is constant or $c_{1} Q^{\prime \prime}\left(\tau_{1}\right)-Q^{\prime \prime}(\tau)=0$. In the second case, we also get $c_{1}=1$. Thus, equation (20) has the form

$$
\tau_{1 x}=\tau_{x}+d\left(\tau, \tau_{1}\right)
$$

Equations of this form were completely classified in [14] (together with their $x$ - and $n$ characteristic rings). It follows from [14] that $Q$ must have the form given in the statement of the lemma.

Returning to the original variable $t$ in equation (20) with $Q$ given by equation (21), we get Theorem 1 .

\subsection{Proof of Theorem 2}

We assume that $f$ is a nonlinear function of $t_{x}$. Thus

$$
f\left(t, t_{1}, t_{x}\right)=A\left(t, t_{1}\right) M\left(t_{x}\right)+B\left(t, t_{1}\right) t_{x}+C\left(t, t_{1}\right)
$$

and equation (1) becomes

$$
t_{1 x}=A\left(t, t_{1}\right) M\left(t_{x}\right)+B\left(t, t_{1}\right) t_{x}+C\left(t, t_{1}\right) .
$$

The proof of the Theorem 2 is based on the following lemmas.

Lemma 9 Let equation (24) have a characteristic n-ring of dimension two, and let $M$ be a nonlinear function. Then the function $M$ satisfies

$$
M^{\prime}=-\frac{\alpha_{2} M+\alpha_{4} t_{x}+\alpha_{6}}{\alpha_{1} M+\alpha_{3} t_{x}+\alpha_{5}},
$$

where $\alpha_{1} M+\alpha_{3} t_{x}+\alpha_{5} \neq 0$. 
Proof If the dimension of the characteristic $n$-ring is two, then $\left(\frac{f_{t}}{f_{t_{x}}}\right)_{t_{1}}=0$. Hence, for $f$ given by equation (23), we have

$$
\begin{aligned}
& \left(\frac{A_{t} M+t_{x} B_{t}+C_{t}}{A M^{\prime}+B}\right)_{t_{1}} \\
& =\frac{\left(A_{t t_{1}} M+t_{x} B_{t t_{1}}+C_{t t_{1}}\right)\left(A M^{\prime}+B\right)-\left(A_{t_{1}} M^{\prime}+B_{t_{1}}\right)\left(A_{t} M+t_{x} B_{t}+C_{t}\right)}{\left(A M^{\prime}+B\right)^{2}}=0 .
\end{aligned}
$$

This can be rewritten as

$$
M^{\prime}\left(\alpha_{1} M+\alpha_{3} t_{x}+\alpha_{5}\right)=-\left(\alpha_{2} M+\alpha_{4} t_{x}+\alpha_{6}\right)
$$

for some constants $\alpha_{i}, i=1,2, \ldots, 5$. Note that if $\alpha_{1} M+\alpha_{3} t_{x}+\alpha_{5}=0$, then either $M^{\prime}=0$ or $\alpha_{2} M+\alpha_{4} t_{x}+\alpha_{6}=0$. In both cases, we get that $f$ is a linear function of $t_{x}$. Hence we can assume that $\alpha_{1} M+\alpha_{3} t_{x}+\alpha_{5} \neq 0$, and we can write equality (25).

The above lemma allows us to express the derivative $M^{\prime}$ in terms of $M$. We can also express the shift $D M$ in terms of $M$. Indeed, as it was proved in [20] (see Lemma 12), if equation (1) has a four-dimensional characteristic $x$-ring and $f_{t_{x} t_{x}} \neq 0$, then

$$
D f=-H_{1}\left(t, t_{1}, t_{2}\right) t_{x}+H_{2}\left(t, t_{1}, t_{2}\right) f+H_{3}\left(t, t_{1}, t_{2}\right)
$$

for some functions $H_{1}, H_{2}$, and $H_{3}$. Therefore,

$$
D\left(A M+B t_{x}+C\right)=-H_{1} t_{x}+H_{2}\left(A M+B t_{x}+\widetilde{C}\right)
$$

and

$$
D M=Q_{1}\left(t, t_{1}, t_{2}\right) M+Q_{2}\left(t, t_{1}, t_{2}\right) t_{x}+Q_{3}\left(t, t_{1}, t_{2}\right)
$$

for some functions $Q_{1}, Q_{2}$ and $Q_{3}$.

We use expressions (25) and (29) for the derivative and shift of $M$ in the next lemma.

Lemma 10 Let equation (24) have a characteristic n-ring of dimension two. Then $M$ has either of the forms $M=\frac{1}{t_{x}+P}$, or $M=\sqrt{t_{x}^{2}+P t_{x}+Q}$, or $M=t_{x}^{2}$.

Proof Consider the vector field $X=\frac{\partial}{\partial t_{x}}$. We can easily check that $D X=\frac{1}{f_{t_{x}}} X D$. Thus $D X(M)=\frac{1}{f_{t_{x}}} X(D M)$. Using equation (25) for $X(M)$ and equation (29) for $D M$, we get

$$
-D\left(\frac{\alpha_{2} M+\alpha_{4} t_{x}+\alpha_{6}}{\alpha_{1} M+\alpha_{3} t_{x}+\alpha_{5}}\right)=\frac{1}{A M^{\prime}+B} X\left(Q_{1} M+Q_{2} t_{x}+Q_{3}\right) .
$$

Using equation (25) and equation (29) once more, we get

$$
\begin{aligned}
&--\tilde{\alpha_{2}}\left(Q_{1} M+Q_{2} t_{x}+Q_{3}\right)+\tilde{\alpha_{4}}\left(A M+B t_{x}+C\right)+\tilde{\alpha_{6}} \\
& \tilde{\alpha_{1}}\left(Q_{1} M+Q_{2} t_{x}+Q_{3}\right)+\tilde{\alpha_{3}}\left(A M+B t_{x}+C\right)+\tilde{\alpha_{5}} \\
&=\frac{Q_{2}-Q_{1} \frac{\alpha_{2} M+\alpha_{4} t_{x}+\alpha_{6}}{\alpha_{1} M+\alpha_{3} x_{x}+\alpha_{5}}}{B-A \frac{\alpha_{2} M+\alpha_{4} t_{x}+\alpha_{6}}{\alpha_{1} M+\alpha_{3} t_{x}+\alpha_{5}}},
\end{aligned}
$$


where $D \alpha_{k}=\tilde{\alpha_{k}}$. Hence we can write

$$
R_{1} M^{2}-\left(R_{2} t_{x}+R_{3}\right) M+\left(R_{4} t_{x}^{2}+R_{5} t_{x}+R_{6}\right)=0
$$

for some functions $R_{k}, k=1,2, \ldots, 6$. Then, we find that

$$
M=\frac{\left(R_{2} t_{x}+R_{3}\right) \pm \sqrt{\left(R_{2} t_{x}+R_{3}\right)^{2}-4 R_{1}\left(R_{4} t_{x}^{2}+R_{5} t_{x}+R_{6}\right)}}{2 R_{1}} \quad \text { if } R_{1} \neq 0
$$

or

$$
M=\frac{R_{4} t_{x}^{2}+R_{5} t_{x}+R_{6}}{R_{2} t_{x}+R_{3}} \quad \text { if } R_{1}=0 .
$$

Since the function $f=A M+B t_{x}+C$ has a linear term $B t_{x}$ and a free term $C$, we can assume that $M$ has the form given in the statement of the lemma.

Now we consider each value of $M$ obtained in the lemma, separately. We start with the simple case $M=t_{x}^{2}$.

Lemma 11 Equation (24) cannot have a four-dimensional characteristic $x$-ring if $M=t_{x}^{2}$.

Proof We can easily check that, for any

$$
f=A\left(t, t_{1}\right) t_{x}^{2}+B\left(t, t_{1}\right) t_{x}+C\left(t, t_{1}\right)
$$

condition (7) is not satisfied. Hence equation (24) cannot have a four-dimensional characteristic $x$-ring if $M=t_{x}^{2}$.

Let us consider the case $M=\frac{1}{t_{x}+P}$.

Lemma 12 Let $M=\frac{1}{t_{x}+P}$, and let equation (24) have a four-dimensional characteristic $x$ ring and a two-dimensional characteristic n-ring. Then equation (24) takes either of the forms

$$
t_{1 x}=\frac{c^{*} \eta(t) \eta\left(t_{1}\right)}{t_{x}} \quad \text { or } \quad t_{1 x}=\frac{c^{*} e^{c^{* *}\left(t+t_{1}\right)}}{t_{x}+P}-P
$$

Proof We have

$$
f\left(t, t_{1}, t_{x}\right)=\frac{A\left(t, t_{1}\right)}{t_{x}+P}+B\left(t, t_{1}\right) t_{x}+C\left(t, t_{1}\right)
$$

Applying condition (7) to $f$, we get

$$
\frac{3\left(t_{x}+P\right)}{B\left(t_{x}+P\right)^{2}+(C+P-B P)\left(t_{x}+P\right)+A}=\frac{3\left(t_{x}+P\right)\left(B\left(t_{x}+P\right)^{2}+A\right)}{\left(B\left(t_{x}+P\right)^{2}-A\right)^{2}} .
$$


From this equality we get

$$
\begin{gathered}
B(C+P-B P)\left(t_{x}+P\right)^{3}+4 A B\left(t_{x}+P\right)^{2} \\
+A(C+P-B P)\left(t_{x}+P\right)=0 .
\end{gathered}
$$

In this equality the coefficients of $\left(t_{x}+P\right)^{k}, k=1,2,3$, must be zero. So we find

$$
B(C+P-B P)=0, \quad 4 A B=0, \quad \text { and } \quad A(C+P-B P)=0 .
$$

Since $A\left(t, t_{1}\right) \neq 0$ (otherwise $f_{t_{x} t_{x}}=0$ ), we find $B=0$ and $C=-P$. Thus we have

$$
f\left(t, t_{1}, t_{x}\right)=\frac{A\left(t, t_{1}\right)}{t_{x}+P}-P .
$$

Using condition (4), we get

$$
\frac{A_{t_{1}}\left(t_{1}, t_{2}\right) A\left(t, t_{1}\right)}{A\left(t_{1}, t_{2}\right)\left(t_{x}+P\right)}=\frac{A_{t_{1}}\left(t, t_{1}\right)}{t_{x}+P}
$$

or

$$
\frac{A_{t_{1}}\left(t_{1}, t_{2}\right)}{A\left(t_{1}, t_{2}\right)}=\frac{A_{t_{1}}\left(t, t_{1}\right)}{A\left(t, t_{1}\right)} .
$$

It follows that $\frac{A_{t_{1}}\left(t_{1}, t_{2}\right)}{A\left(t_{1}, t_{2}\right)}$ does not depend on $t_{2}$, so $\frac{\partial^{2}}{\partial t_{2} \partial t_{1}} \ln A\left(t_{1}, t_{2}\right)=0$, and $\frac{A_{t_{1}}\left(t, t_{1}\right)}{A\left(t, t_{1}\right)}$ does not depend on $t$, so $\frac{\partial^{2}}{\partial t \partial t_{1}} \ln A\left(t, t_{1}\right)=0$.

Hence we get $A\left(t, t_{1}\right)=\varphi(t) \eta\left(t_{1}\right)$ for some functions $\varphi$ and $\eta$. Using equation (34), we obtain $\frac{\varphi^{\prime}\left(t_{1}\right)}{\varphi\left(t_{1}\right)}=\frac{\eta^{\prime}\left(t_{1}\right)}{\eta\left(t_{1}\right)}$, which implies that $\varphi\left(t_{1}\right)=c^{*} \eta\left(t_{1}\right)$, where $c^{*}$ is some constant. Hence we have

$$
f=\frac{c^{*} \eta(t) \eta\left(t_{1}\right)}{t_{x}+P}-P .
$$

From condition (8) it follows that

$$
\tilde{m}=\left(-\frac{P \eta^{\prime}(t)}{2 \eta(t)}+\frac{P \eta^{\prime}\left(t_{1}\right)}{2 \eta\left(t_{1}\right)}\right)\left(t_{x}+P\right)-\frac{\left(t_{x}+P\right)^{2} \eta^{\prime}(t)}{2 \eta(t)}
$$

does not depend on $t_{1}$. So, either $P=0$ or $\eta^{\prime}\left(t_{1}\right)=c^{* *} \eta\left(t_{1}\right)$, which implies $\eta\left(t_{1}\right)=e^{c^{* *}} t_{1}$ with some constant $c^{* *}$. Thus we obtain equations (31). We can easily check that these equations have a two-dimensional characteristic $n$-ring and a four-dimensional characteristic $x$-ring.

Let us consider the case $M=\sqrt{t_{x}^{2}+P t_{x}+Q}$.

Lemma 13 Let $M=\sqrt{t_{x}^{2}+P t_{x}+Q}$, and let equation (24) have a four-dimensional characteristic $x$-ring and a two-dimensional characteristic n-ring. Then equation (24) takes the form

$$
t_{1 x}=\sqrt{B^{2}-1} \sqrt{t_{x}^{2}+P t_{x}+Q}+B t_{x}+\frac{1}{2} P(B-1),
$$

where $B, Q, P$ are constants, and $B^{2} \neq 1$. 
Proof We have

$$
f=A\left(t, t_{1}\right) \sqrt{t_{x}^{2}+P t_{x}+Q}+B\left(t, t_{1}\right) t_{x}+C\left(t, t_{1}\right)
$$

Applying condition (7), we find

$$
\begin{aligned}
- & \frac{6 B P+12 t_{x}+12 A \sqrt{t_{x}^{2}+t_{x} P+Q}}{\left(A P+2 A t_{x}+2 B \sqrt{t_{x}^{2}+t_{x} P+Q}\right)^{2}} \\
& =\frac{-3 P-6 C-6 B t_{x}-6 A \sqrt{t_{x}^{2}+P t_{x}+Q}}{2 Q+2\left(C+B t_{x}+A \sqrt{t_{x}^{2}+P t_{x}+Q}\right)\left(C+P+B t_{x}+A \sqrt{t_{x}^{2}+P t_{x}+Q}\right)}
\end{aligned}
$$

or

$$
\begin{aligned}
4(Q+ & \left.\left(C+B t_{x}+A \sqrt{t_{x}^{2}+P t_{x}+Q}\right)\left(C+P+B t_{x}+A \sqrt{t_{x}^{2}+P t_{x}+Q}\right)\right) \\
& \cdot\left(B P+2 t_{x}+2 A \sqrt{t_{x}^{2}+t_{x} P+Q}\right) \\
= & \left(A P+2 A t_{x}+2 B \sqrt{t_{x}^{2}+t_{x} P+Q}\right)^{2}\left(P+2 C+2 B t_{x}+2 A \sqrt{t_{x}^{2}+P t_{x}+Q}\right) .
\end{aligned}
$$

Comparing the coefficients of $\left(\sqrt{t_{x}^{2}+P t_{x}+Q}\right)^{i}\left(t_{x}\right)^{j}$ for $i, j=0,1,2$, we get

$$
\begin{aligned}
& A B(2 C+P-B P)=0, \\
& A\left(-4 C^{2}-4 C P+A^{2}\left(P^{2}-4 Q\right)+4\left(B^{2}-1\right) Q\right)=0, \\
& \left(A^{2}+B^{2}\right)(-2 C+(B-1) P)=0, \\
& -2 A^{2} P(2 C+P)+4 B^{3} Q-B\left(4 C^{2}+4 C P+4 Q+A^{2}\left(-3 P^{2}+4 Q\right)\right)=0, \\
& A^{2}(2 C+P)\left(P^{2}-8 Q\right)+4 B^{2}(2 C+P) Q-4 B P\left(C^{2}+C P+Q-A^{2} Q\right)=0 .
\end{aligned}
$$

We can check that these equalities are satisfied if and only if

$$
2 C=P B-P \quad \text { and } \quad 4 C^{2}+4 C P=A^{2}\left(P^{2}-4 Q\right)-4 Q+4 B^{2} Q .
$$

Simplifying, we get

$$
2 C=P B-P, \quad \text { and either } \quad B^{2}=A^{2}+1 \quad \text { or } \quad P^{2}=4 Q .
$$

In the case $P^{2}=4 Q$, we have that $M=\sqrt{t_{x}^{2}+P t_{x}+Q}$ is a linear function of $t_{x}$. Therefore we have to study only the case $B^{2}=A^{2}+1$. Thus we have

$$
f=\sqrt{B\left(t, t_{1}\right)^{2}-1} \sqrt{t_{x}^{2}+P t_{x}+Q}+B\left(t, t_{1}\right) t_{x}+\frac{P}{2}\left(B\left(t, t_{1}\right)-1\right)
$$

where $B \neq 1$. In the same way, we check that condition (4) in the form $\left(D \frac{f_{t}}{f_{t_{x}}}\right)^{2}-\left(f_{t_{1}}\right)^{2}=0$ is satisfied for this function $f$ if and only if

$$
\frac{B_{t_{1}}^{2}\left(t, t_{1}\right)}{B^{2}\left(t, t_{1}\right)-1}=\frac{B_{t_{1}}^{2}\left(t_{1}, t_{2}\right)}{B^{2}\left(t_{1}, t_{2}\right)-1}
$$


Hence we can write

$$
B_{t_{1}}\left(t, t_{1}\right)=A\left(t, t_{1}\right) \varphi\left(t_{1}\right), B_{t}\left(t, t_{1}\right)= \pm A\left(t, t_{1}\right) \varphi(t)
$$

for some function $\varphi$.

Using condition (8), let us show that $B$ can only be a constant function. We have

$$
\tilde{m}=\mu_{1} t_{x}\left(t_{x}^{2}+p t_{x}+q\right)+\mu_{2}\left(t_{x}^{2}+p t_{x}+q\right)^{\frac{3}{2}}+\mu_{3}\left(t_{x}^{2}+p t_{x}+q\right)
$$

where

$$
\begin{aligned}
& \mu_{1}=\frac{2 P B\left(B_{t_{1}}+B_{t}\right)}{\left(P^{2}-4 Q\right)\left(B^{2}-1\right)}, \\
& \mu_{2}=\frac{2 P \sqrt{B^{2}-1}\left(B_{t_{1}}+B_{t}\right)}{\left(P^{2}-4 Q\right)\left(B^{2}-1\right)}, \\
& \mu_{3}=\frac{\left(4 Q-P^{2}+P^{2} B\right) B_{t_{1}}+4 Q B B_{t}}{\left(P^{2}-4 Q\right)\left(B^{2}-1\right)} .
\end{aligned}
$$

Since $\tilde{m}$ does not depend on $t_{1}$, we have that $\mu_{1}, \mu_{2}$, and $\mu_{3}$ also do not depend on $t_{1}$. Using (41), we have

$$
\mu_{2}=\frac{2 P\left(\varphi\left(t_{1}\right) \pm \varphi(t)\right)}{P^{2}-4 Q} .
$$

Since $\mu_{2}$ does not depend on $t_{1}$, either $\varphi$ is a constant function or $P=0$. Note that in both cases, we get $\mu_{1}=0$ and $\mu_{2}=0$. We start with the case where $\phi$ is some constant $C$. Using equation (41), we have

$$
\mu_{3}=\frac{C\left(4 Q-P^{2}\right)+C\left(P^{2} \pm 4 Q\right) B}{\left(P^{2}-4 Q\right) \sqrt{B^{2}-1}} .
$$

Differentiating this equality with respect to $t_{1}$, we get

$$
0=\frac{C B_{t_{1}}\left(\left(P^{2} \pm 4 Q\right)+\left(4 Q-P^{2}\right) B\right)}{\left(P^{2}-4 Q\right)\left(B^{2}-1\right)^{\frac{3}{2}}},
$$

which gives $B_{t_{1}}=0$ or $\left(\left(P^{2} \pm 4 Q\right)+\left(4 Q-P^{2}\right) B\right)=0$. Both equalities imply that $B$ is a constant.

Now we consider the case $P=0$. Then, using equation (41), we have

$$
\mu_{3}=\frac{4 Q\left(B_{t_{1}}+B B_{t}\right)}{-4 Q\left(B^{2}-1\right)}=-\frac{\varphi\left(t_{1}\right) \pm B \varphi(t)}{\sqrt{B^{2}-1}} .
$$

Differentiating this equality with respect to $t_{1}$, we get

$$
0=\frac{B_{t_{1}}\left(B \varphi\left(t_{1}\right) \pm \varphi(t)\right)}{\left(B^{2}-1\right)^{\frac{3}{2}}},
$$

which implies that either $B_{t_{1}}=0$ or $B= \pm \frac{\varphi(t)}{\varphi\left(t_{1}\right)}$. In both cases, we get that $B$ is a constant function. Indeed, if $B_{t_{1}}=0$, then $B_{t}=0$ by equation (41), so $B$ is a constant function, and if 
$B= \pm \frac{\phi(t)}{\phi\left(t_{1}\right)}$, then $\mu_{3}= \pm \sqrt{\varphi^{2}(t)-\varphi^{2}\left(t_{1}\right)}$, and since $\mu_{3}$ does not depend on $t_{1}$, we get that $\phi$ is a constant function, and hence $B$ is a constant function. Using the equality $B^{2}=A^{2}+1$, we get the statement of the lemma.

The proof of Theorem 2 easily follows from the above lemmas.

\section{Examples}

The functions $f$ given in the Theorem 1 lead to the following examples.

Example 14 The equation

$$
t_{1 x}=\frac{\gamma(t)}{\gamma\left(t_{1}\right)} t_{x}-\frac{\gamma(t)}{\gamma\left(t_{1}\right)} \sigma(t)+\sigma\left(t_{1}\right)
$$

where functions $\gamma$ and $\sigma$ satisfy the relation

$$
(\gamma(t) \sigma(t))^{\prime}=\gamma(t) \sqrt{B_{1}+B_{2}(\gamma(t) \sigma(t))}, \quad B_{1}, B_{2} \in \mathbb{R}
$$

has an $x$-integral $F=\frac{\left(L\left(t_{3}\right)-L\left(t_{1}\right)\right)\left(L\left(t_{2}\right)-L(t)\right)}{\left(L\left(t_{3}\right)-L\left(t_{2}\right)\right)\left(L\left(t_{2}\right)-L\left(t_{1}\right)\right)}$, where $L(t)=\int_{0}^{t} \gamma(\tau) d \tau$, and an $n$-integral $I=$ $\gamma(t) t_{x}-\sigma(t)$.

Example 15 The equation

$$
t_{1 x}=\frac{\gamma(t)}{\gamma\left(t_{1}\right)} t_{x}-\frac{\gamma(t)}{\gamma\left(t_{1}\right)} \sigma(t)+\sigma\left(t_{1}\right),
$$

where functions $\gamma$ and $\sigma$ satisfy the relation

$$
(\gamma(t) \sigma(t))^{\prime}=\gamma(t) \sqrt{B_{1}+B_{2}(\gamma(t) \sigma(t))^{2}}, \quad B_{1}, B_{2} \in \mathbb{R}
$$

has an $x$-integral $F=\frac{\left(e^{L(t)}-e^{L\left(t_{2}\right)}\right)\left(e^{L\left(t_{1}\right)}-e^{L\left(t_{3}\right)}\right)}{\left(e^{L(t)}-e^{L\left(t_{3}\right)}\right)\left(e^{L\left(t_{1}\right)}-e^{L\left(t_{2}\right)}\right)}$, where $L(t)=\int_{0}^{t} \gamma(\tau) d \tau$, and an $n$-integral $I=$ $\gamma(t) t_{x}-\sigma(t)$.

The functions $f$ given in the Theorem 2 lead to the following examples.

Example 16 The equation

$$
t_{1 x}=\frac{c_{1} \eta(t) \eta\left(t_{1}\right)}{t_{x}}
$$

has an $x$-integral $F=\int_{0}^{t_{3}} \eta^{-1}(\tau) d \tau-\int_{0}^{t_{1}} \eta^{-1}(\tau) d \tau$ and an $n$-integral $I=\frac{t_{x}}{c_{1} \eta(t)}+\frac{\eta(t)}{t_{x}}$.

Example 17 The equation

$$
t_{1 x}=\frac{c_{1} e^{c_{2}\left(t+t_{1}\right)}}{t_{x}+P}-P
$$

has an $x$-integral $F=e^{-c_{2} t_{3}+c_{2} P x}-e^{-c_{2} t_{1}+c_{2} P x}$ and an $n$-integral $I=\frac{t_{x}+P}{c_{1} e^{c_{2} t}}+\frac{e^{c_{2} t}}{t_{x}+P}$. 
Example 18 The equation

$$
t_{1 x}=\sqrt{B^{2}-1} \sqrt{t_{x}^{2}+P t_{x}+Q}+B t_{x}+\frac{1}{2} P(B-1)
$$

has an $x$-integral

$$
F=\left(-8 B^{3}-4 B^{2}+4 B-1\right) t+\left(8 B^{3}-2 B+1\right) t_{1}+\left(-4 B^{2}+2 B-1\right) t_{2}+t_{3}
$$

and an $n$-integral

$$
I=\left(B-\sqrt{B^{2}-1}\right)^{n}\left(\sqrt{t_{x}^{2}+P t_{x}+Q}+t_{x}+0.5 P\right) .
$$

In all examples, we can check that $F$ is an $x$-integral and $I$ is an $n$-integral by direct calculations.

\section{Competing interests}

The authors declare that they have no competing interests.

\section{Authors' contributions}

All authors contributed equally to this work. All authors read and approved the final manuscript.

\section{Author details}

'Department of Mathematics, Middle East Technical University, Universiteler Mahallesi, Dumlupinar Bulvari 1, Ankara, 06800, Turkey. ${ }^{2}$ Department of Mathematics, Bilkent University, Bilkent, Ankara, 06800, Turkey.

\section{Publisher's Note}

Springer Nature remains neutral with regard to jurisdictional claims in published maps and institutional affiliations.

Received: 27 January 2017 Accepted: 13 June 2017 Published online: 27 June 2017

\section{References}

1. Shabat, AB, Yamilov, RI: Exponential systems of type I and Cartan matrices. Preprint BBAS USSR Ufa (1981) (in Russian)

2. Leznov, AN, Smirnov, VG, Shabat, AB: Internal symmetry group and integrability conditions for two-dimensional dynamical systems. Teor. Mat. Fiz. 51, 10-21 (1982) (in Russian)

3. Sokolov, WV, Zhiber, AV: On the Darboux integrable hyperbolic equations. Phys. Lett. A 208, 303-308 (1995)

4. Zhiber, AV, Sokolov, VV, Ya, SS: On nonlinear Darboux-integrable hyperbolic equations. Dokl. Akad. Nauk, Ross. Akad. Nauk 343, 746-748 (1995) (in Russian)

5. Zhiber, AV, Sokolov, V: Exactly integrable hyperbolic equations of Liouville type. Russ. Math. Surv. 56, 61-101 (2001)

6. Zhiber, AV, Murtazina, RD: On the characteristic Lie algebras for the equations $u_{x y}=f\left(u, u_{x}\right)$. J. Math. Sci. (N.Y.) 151, 3112-3122 (2008)

7. Kostrigina, OS, Zhiber, AV: Darboux-integrable two-component nonlinear hyperbolic systems of equations. J. Math. Phys. 52, 033503 (2011)

8. Habibullin, IT: Characteristic algebras of fully discrete hyperbolic type equations. SIGMA 1, 023 (2005)

9. Habibullin, IT, Pekcan, A: Characteristic Lie algebra and the classification of semi-discrete models. Theor. Math. Phys. $151,781-790(2007)$

10. Habibullin, IT: Characteristic algebras of discrete equations. In: Difference Equations, Special Functions and Orthogonal Polynomials, pp. 249-257. World Scientific, Hackensack (2007)

11. Habibullin, IT, Gudkova, EV: Classification of integrable discrete Klein-Gordon models. Phys. Scr. 81, 045003 (2011)

12. Habibullin, IT, Zheltukhina, N, Pekcan, A: On some algebraic properties of semi-discrete hyperbolic type equations. Turk. J. Math. 32, 277-292 (2008)

13. Habibullin, IT, Zheltukhina, N, Pekcan, A: On the classification of Darboux integrable chains. J. Math. Phys. 49, 102702 (2008)

14. Habibullin, IT, Zheltukhina, N, Pekcan, A: Complete list of Darboux integrable chains of the form $t_{1 x}=t_{x}+d\left(t, t_{1}\right)$. J. Math. Phys. 50, 102710 (2009)

15. Habibullin, IT, Zheltukhina, N, Sakieva, A: On Darboux-integrable semi-discrete chains. J. Phys. A 43, 434017 (2010)

16. Habibullin, IT, Zheltukhina, N: Discretization of Liouville type nonautonomous equations. J. Nonlinear Math. Phys. 23, 620-642 (2016)

17. Zhiber, AB, Murtazina, RD, Habibullin, IT, Shabat, AB: Characteristic Lie rings and integrable models in mathematical physics. Ufa Math. J. 4, 17-85 (2012)

18. Darboux, G: Leçons sur la théorie générale des surfaces et les applications géométriques du calculus infinitésimal, vol. 2. Gautier Villas, Paris (1915) 
19. Adler, VE, Ya, SS: Discrete analogues of the Liouville equation. Theor. Math. Phys. 121, 1484 (1999)

20. Zheltukhin, $\mathrm{K}$, Zheltukhina, N: On existence of an $\mathrm{x}$-integral for a semi-discrete chain of hyperbolic type. J. Phys. Conf. Ser. 670, 434017 (2016)

21. Zheltukhin, K, Zheltukhina, N: Semi-discrete hyperbolic equations admitting five dimensional characteristic $x$-ring J. Nonlinear Math. Phys. 23, 351-367 (2016)

Submit your manuscript to a SpringerOpen ${ }^{\circ}$ journal and benefit from:

- Convenient online submission

$\checkmark$ Rigorous peer review

- Open access: articles freely available online

- High visibility within the field

- Retaining the copyright to your article

Submit your next manuscript at $\gg$ springeropen.com 